\title{
Circular phase-dichroism of chiral metasurface using
}

\section{birefringent interference}

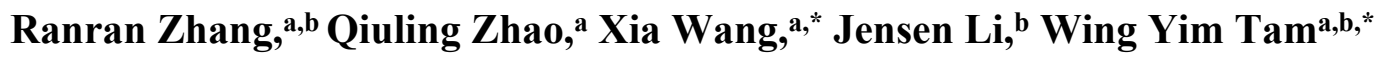 \\ ${ }^{a}$ Optoelectronic Materials and Technologies Engineering Laboratory, Shandong, Physics \\ Department, QingDao University of Science and Technology, China \\ ${ }^{b}$ Department of Physics, William Mong Institute of Nano Science and Technology, and Center \\ for Metamaterial Research, Hong Kong University of Science and Technology, Clear Water Bay, \\ Kowloon, Hong Kong, China \\ *E-mail address for correspondence: phwangxia@163.com,phtam@ust.hk
}

\section{Supporting Information}

\section{Fabrication of Au sawtooth nanoarray metasurfaces}

A $30 \mathrm{~nm}$ thick gold was first deposited onto a $1 \times 1 \mathrm{~cm}^{2} 2 \mathrm{~mm}$ thick a-cut sapphire substrate using e-beam evaporation. Then a focused ion-beam was used to direct write $50 \times 50 \mu \mathrm{m}^{2} \mathbf{V}$-, $\mathbf{N}$ - and $\boldsymbol{U}$-type sawtooth nanohole array patterns with 450nm lattice spacing in both $\hat{x}$ and $\hat{y}$ directions. The patterns were written with the horizontal direction along the optical axis $(\hat{x}-$ axis) of the sapphire. The Au sawtooth width can be varied by controlling the i-beam dosage. (To fabricate metasurfaces with non-birefringent substrates, e.g. on ITO glass, we used e-beam direct-write and lift-off techniques.)

\section{Transmission measurement}

Figure S1 shows the setup for the transmission measurement of metasurfaces. We use a 100W halogen lamp of a microscope as the light source. The light first passes through a polarizer (P1) and then a super-achromatic zero-order quarter waveplate (Q1, APSAW-5 from Astropribor) to produce either a linearly $\left(\alpha=0^{\circ}\right.$ or $\left.90^{\circ}\right)$ or circularly polarized incident light ( $\alpha$ 
$=-/+45^{\circ}$ for LCP/RCP) with wavelengths from $450-850 \mathrm{~nm}$ [1]. The incident light enters the sample from the substrate side (sapphire) and then passes through the metasurface. The transmitted light is then measured using a Stokes setup [2] consisting of another zero-order quarter waveplate $(\mathrm{Q} 2)$ and a polarizer $(\mathrm{P} 2)$. Detection of $\mathrm{LCP} / \mathrm{RCP}$ transmission can be achieved by setting the angle $\beta$ to $+/-45^{\circ}$, respectively. We use a $20 \mathrm{X}$ objective to collect the transmitted light by focusing the objective onto a $25 \mathrm{x} 25 \mu \mathrm{m}^{2}$ area, small enough to cover our Au sawtooth metasurface of size $50 \times 50 \mu \mathrm{m}^{2}$. The collected light is then coupled through an optical fiber to an Ocean Optics S4000 spectrometer for spectral measurements.

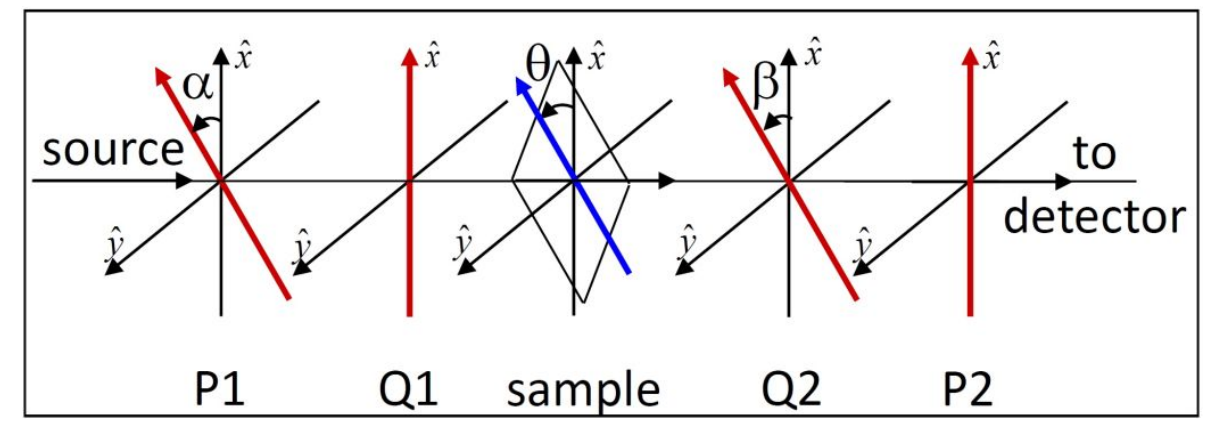

Figure S1. Schematic setup for transmission measurement. P1 and P2 are polarizers and Q1 and Q2 are quarter waveplates. The red arrows are the polarizer/fast axes ( $\hat{x}$-redirection) of the polarizers/waveplates. LCP/RCP incidence is obtained by setting $\alpha=-/+45^{\circ}$ and detection of $\mathrm{LCP} / \mathrm{RCP}$ by $\beta=+/-45^{\circ}$. $\theta$ is the angle between the optical axis of the sapphire (blue arrow) and the reference $\hat{x}$-axis.

\section{Transmission of metasurface using Jones Matrix}

We use the Jones matrix approach to model the transmission of our 2D Au sawtooth metasurface. We choose the $\hat{z}$-axis as the propagation direction with $\boldsymbol{E}$ field amplitude written as:

$$
\boldsymbol{E}=\left(\begin{array}{c}
E_{x}(t) \\
E_{y}(t) \\
0
\end{array}\right)=\left(\begin{array}{c}
E_{0 x} e^{i \phi_{x}} \\
E_{0 y} e^{i \phi_{y}} \\
0
\end{array}\right) e^{i(k z-\omega t)}
$$


where $E_{0 x / 0 y}$ is the amplitude and $\phi_{x / y}$ is the initial phase delay. $k=2 \pi / \lambda$ is the wave vector with $\lambda$ the wavelength; $\omega$ is the angular frequency. For uniaxial birefringent crystal, the refractive index for the E-field component along the extraordinary (optical) axis $\left(n_{e}\right)$ is different from that of the ordinary axis $\left(n_{o}\right)$. Furthermore, for an a-cut sapphire slab, the extraordinary axis is on the plane of the surface as indicated by the blue arrow in Fig. S1 [3]. For Jones representation, the transfer matrix for the sapphire for normal incidence in circular polarization bases can be written as $[4,5]$ :

$$
\boldsymbol{t}^{S a}=\left(\begin{array}{cc}
t_{+++}^{S a} & t_{+-}^{S a} \\
t_{-+}^{S a} & t_{--}^{S a}
\end{array}\right)=\left(\begin{array}{cc}
\cos \left(\frac{\delta}{2}\right) & i e^{-i 2 \theta} \sin \left(\frac{\delta}{2}\right) \\
i e^{i 2 \theta} \sin \left(\frac{\delta}{2}\right) & \cos \left(\frac{\delta}{2}\right)
\end{array}\right)
$$

where $\delta=\frac{2 \pi}{\lambda}\left(n_{o}-n_{e}\right) L$ for $L$ the thickness of the sapphire and $\theta$ is the angle between the optical axis of the sapphire and the reference $\hat{x}$-axis. We choose $+/$ - to denote right/left-handed circularly polarization (RCP/LCP), respectively. (The subscript indexes, $i$ and $j$, of $t_{i j}^{S a}$ are the outgoing and incoming rays, respectively.) Thus, the transmission coefficients for RCP $\left(\begin{array}{l}1 \\ 0\end{array}\right)$ and LCP $\left(\begin{array}{l}0 \\ 1\end{array}\right)$ incidence can be expressed as:

$$
\left(\begin{array}{c}
E_{+++}^{S a} \\
E_{-+}^{S a}
\end{array}\right)=\boldsymbol{t}^{S a}\left(\begin{array}{l}
1 \\
0
\end{array}\right)=\left(\begin{array}{c}
\cos \left(\frac{\delta}{2}\right) \\
i e^{i 2 \theta} \sin \left(\frac{\delta}{2}\right)
\end{array}\right),
$$

and

$$
\left(\begin{array}{l}
E_{+-}^{S a} \\
E_{--}^{S a}
\end{array}\right)=\boldsymbol{t}^{S a}\left(\begin{array}{l}
0 \\
1
\end{array}\right)=\left(\begin{array}{c}
i e^{-i 2 \theta} \sin \left(\frac{\delta}{2}\right) \\
\cos \left(\frac{\delta}{2}\right)
\end{array}\right),
$$

respectively.

The transmittance through the sapphire plate can then be written as: 


$$
\begin{gathered}
T_{++}^{S a}=E_{++}^{S a} * E_{++}^{S a *}=\cos \left(\frac{\delta}{2}\right) * \cos \left(\frac{\delta}{2}\right)=\frac{1}{2}(1+\cos \delta), \\
T_{--}^{S a}=E_{--}^{S a} * E_{--}^{S a *}=\cos \left(\frac{\delta}{2}\right) * \cos \left(\frac{\delta}{2}\right)=\frac{1}{2}(1+\cos \delta) . \\
T_{+-}^{S a}=E_{+-}^{S a} * E_{+-}^{S a *}=i e^{-i 2 \theta} \sin \left(\frac{\delta}{2}\right) *\left(-i e^{i 2 \theta} \sin \left(\frac{\delta}{2}\right)\right)=\frac{1}{2}(1+\cos (\delta \pm \pi)),(\mathrm{S} 4 \mathrm{c}) \\
T_{-+}^{S a}=E_{-+}^{S a} * E_{-+}^{S a *}=i e^{i 2 \theta} \sin \left(\frac{\delta}{2}\right) *\left(-i e^{-i 2 \theta} \sin \left(\frac{\delta}{2}\right)\right)=\frac{1}{2}(1+\cos (\delta \pm \pi)),(\mathrm{S} 4 \mathrm{~d})
\end{gathered}
$$

Note that as $\delta$ (or wavenumber $1 / \lambda$ ) varies with a period inversely proportional to $L$ all transmittance exhibit birefringent interference $(\mathrm{BI})$ oscillations similar to those oscillations due to the Fabry-Perot interference [6, 7]. Moreover, these BI oscillations are independent of $\theta$, and $T_{++}^{S a}=T_{--}^{S a}$ is $\pi$ out of phase with $T_{+-}^{S a}=T_{-+}^{S a}$.

Using the same approach, the Jones matrix for the metasurface can be written as:

$$
\boldsymbol{t}^{l}=\left(\begin{array}{ll}
t_{++}^{l} & t_{+-}^{l_{+}} \\
t_{-+}^{l} & t_{--}^{l}
\end{array}\right)
$$

where $l=\mathrm{Au}, V, N$, and $N^{-1}$ denote the Au film, and the $\mathbf{V}$-, $\mathbf{N}$ - and $\mathbf{U}$-type 2D Au sawtooth metasurfaces, respectively. Thus, the transmission coefficients for the metasurface on a sapphire substrate with incidence from the sapphire side can be expressed as:

$$
\left(\begin{array}{l}
E_{++}^{l} \\
E_{-+}^{l}
\end{array}\right)=\left(\begin{array}{ll}
t_{++}^{l} & t_{+-}^{l} \\
t_{-+}^{l} & t_{--}^{l}
\end{array}\right)\left(\begin{array}{cc}
\cos \left(\frac{\delta}{2}\right) & i e^{-i 2 \theta} \sin \left(\frac{\delta}{2}\right) \\
i e^{i 2 \theta} \sin \left(\frac{\delta}{2}\right) & \cos \left(\frac{\delta}{2}\right)
\end{array}\right)\left(\begin{array}{l}
1 \\
0
\end{array}\right)
$$




$$
\begin{aligned}
& =\left(\begin{array}{l}
/ t_{++}^{l} / e^{i \arg \left(t_{++}^{l}\right)} \cos \left(\frac{\delta}{2}\right)+i / t_{+-}^{l} / e^{i\left[\arg \left(t_{+-}^{l}\right)+2 \theta\right]} \sin \left(\frac{\delta}{2}\right) \\
/ t_{-+}^{l} / e^{i \arg \left(t_{-+}^{l}\right)} \cos \left(\frac{\delta}{2}\right)+i / t_{--}^{l} / e^{i\left[\arg \left(t_{--}^{l}\right)+2 \theta\right]} \sin \left(\frac{\delta}{2}\right)
\end{array}\right), \\
& \left(\begin{array}{l}
E_{+-}^{l} \\
E_{--}^{l}
\end{array}\right)=\left(\begin{array}{ll}
t_{++}^{l} & t_{+-}^{l} \\
t_{-+}^{l} & t_{--}^{l}
\end{array}\right)\left(\begin{array}{cc}
\cos \left(\frac{\delta}{2}\right) & i e^{-i 2 \theta} \sin \left(\frac{\delta}{2}\right) \\
i e^{i 2 \theta} \sin \left(\frac{\delta}{2}\right) & \cos \left(\frac{\delta}{2}\right)
\end{array}\right)\left(\begin{array}{l}
0 \\
1
\end{array}\right) \\
& =\left(\begin{array}{l}
i / t_{++}^{l} / e^{i\left[\arg \left(t_{++}^{l}\right)-2 \theta\right]} \sin \left(\frac{\delta}{2}\right)+/ t_{+-}^{l} / e^{i \arg \left(t_{+-}^{l}\right)} \cos \left(\frac{\delta}{2}\right) \\
i / t_{-+}^{l} / e^{i\left[\arg \left(t_{-+}^{l}\right)-2 \theta\right]} \sin \left(\frac{\delta}{2}\right)+/ t_{--}^{l} / e^{i \arg \left(t_{--}^{l}\right)} \cos \left(\frac{\delta}{2}\right)
\end{array}\right) .
\end{aligned}
$$

Furthermore, the transmittance can be written as:

$$
\begin{aligned}
& T_{++}^{l}=E_{++}^{l} * E_{++}^{l *} \\
& =\frac{1}{2}\left\{/ t_{+-}^{l} /^{2}+/ t_{++}^{l} /^{2}+\left(/ t_{++}^{l} / /^{2}-/ t_{+-}^{l} /^{2}\right) \cos \delta-2 / t_{++}^{l} / / t_{+-}^{l} /\right. \\
& \left.\sin \left[\arg \left(t_{+-}^{l}\right)-\arg \left(t_{++}^{l}\right)+2 \theta\right] \sin \delta\right\} \\
& =\frac{1}{2}\left\{A_{1}+C_{1} \cos \delta-C_{2} \sin \delta\right\}=\frac{1}{2}\left\{A_{1}+\sqrt{C_{1}^{2}+C_{2}^{2}} \cos \left(\delta+\phi_{1}\right)\right\}, \\
& T_{--}^{l}=E_{--}^{l} * E_{--}^{l *} \\
& =\frac{1}{2}\left\{/ t_{--}^{l} /^{2}+/ t_{-+}^{l} /^{2}+\left(/ t_{--}^{l} /^{2}-/ t_{-+}^{l} /^{2}\right) \cos \delta+2 / t_{--}^{l} / / t_{-+}^{l} /\right. \\
& \left.\sin \left[\arg \left(t_{--}^{l}\right)-\arg \left(t_{-+}^{l}\right)+2 \theta\right] \sin \delta\right\} \\
& =\frac{1}{2}\left\{A_{2}+C_{3} \cos \delta-C_{4} \sin \delta\right\}=\frac{1}{2}\left\{A_{2}+\sqrt{C_{3}{ }^{2}+C_{4}{ }^{2}} \cos \left(\delta+\phi_{2}\right)\right\} \text {, } \\
& T_{+-}^{l}=E_{+-}^{l} * E_{+-}^{l *} \\
& =\frac{1}{2}\left\{/ t_{+-}^{l} /^{2}+/ t_{++}^{l} /^{2}+\left(/ t_{+-}^{l} /^{2}-/ t_{++}^{l} /^{2}\right) \cos \delta+2 / t_{+-}^{l} / / t_{++}^{l} /\right. \\
& \left.\sin \left[\arg \left(t_{+-}^{l}\right)-\arg \left(t_{++}^{l}\right)+2 \theta\right] \sin \delta\right\} \\
& =\frac{1}{2}\left\{A_{1}-C_{1} \cos \delta+C_{2} \sin \delta\right\}=\frac{1}{2}\left\{A_{1}+{\sqrt{C_{1}{ }^{2}+C_{2}}}^{2} \cos \left(\delta+\phi_{1}+\pi\right)\right\}, \\
& T_{-+}^{l}=E_{-+}^{l} * E_{-+}^{l *}
\end{aligned}
$$




$$
\begin{gathered}
=\frac{1}{2}\left\{/ t_{--}^{l} /^{2}+/ t_{-+}^{l} /^{2}+\left(/ t_{-+}^{l} /^{2}-/ t_{--}^{l} /^{2}\right) \cos \delta-2 / t_{--}^{l} / / t_{-+}^{l} /\right. \\
\left.\sin \left[\arg \left(t_{--}^{l}\right)-\arg \left(t_{-+}^{l}\right)+2 \theta\right] \sin \delta\right\} \\
=\frac{1}{2}\left\{A_{2}-C_{3} \cos \delta+C_{4} \sin \delta\right\}=\frac{1}{2}\left\{A_{2}+\sqrt{C_{3}^{2}+C_{4}^{2}} \cos \left(\delta+\phi_{2}+\pi\right)\right\}, \quad(\mathrm{S} 7 \mathrm{~d})
\end{gathered}
$$

for

$$
\left\{\begin{array}{c}
A_{1}=/ t_{++}^{l} /^{2}+/ t_{+-}^{l} /^{2} \\
A_{2}=/ t_{--}^{l} /^{2}+/ t_{-+}^{l} /^{2} \\
C_{1}=/ t_{++}^{l} /^{2}-/ t_{+-}^{l} /^{2} \\
C_{2}=2 / t_{++}^{l} / / t_{+-}^{l} / \sin \left[\arg \left(t_{+-}^{l}\right)-\arg \left(t_{++}^{l}\right)+2 \theta\right] \\
C_{3}=/ t_{--}^{l} /^{2}-/ t_{-+}^{l} /^{2} \\
C_{4}=2 / t_{--}^{l} / / t_{-+}^{l} / \sin \left[\arg ^{l}\left(t_{-+}\right)-\arg \left(t_{--}^{l}\right)-2 \theta\right] \\
\phi_{1}=\tan ^{-1} \frac{C_{2}}{C_{1}} \\
\phi_{2}=\tan ^{-1} \frac{C_{4}}{C_{3}}
\end{array}\right.
$$

Note that all the transmittance of the metasurface with a sapphire substrate exhibit similar BI oscillations as that of the sapphire plate except that they are now $\theta$ dependent. Thus, for simplicity, we choose $\theta=0$ for the experiments and simulations. Furthermore, it is clear from Eqs. S4 and S7 that the transmission BI oscillations for the metasurface on sapphire have a phase shift, $\phi_{1}$ or $\phi_{2}$, w.r.t. to that of the sapphire substrate. As $\phi_{1}$ and $\phi_{2}$ are related to the transmission coefficient $t_{i j}^{l}$ for the metasurface as shown in Eq. S8, the chiral property of the metasurface can then be characterized simply by measuring the phase shift w.r.t. that of the sapphire or in a more general term - circular phase-dichroism (CPD) defined as:

$$
\Delta \phi_{c o}^{l}=\Delta \phi_{++}^{l}-\Delta \phi_{--}^{l}=\phi_{1}-\phi_{2}
$$

and

$$
\Delta \phi_{\text {cross }}^{l}=\Delta \phi_{+-}^{l}-\Delta \phi_{-+}^{l}=\phi_{1}-\phi_{2}
$$


for the co- and cross-polarization incidence and detection, respectively. Note that the circular phase-dichroism $\Delta \phi_{c o}\left(=\Delta \phi_{\text {cross }}\right)$ does not depend on the thickness of the sapphire, as it is a property of the metasurface and should be independent of the thickness of the substrate.

\section{Transmission phase}

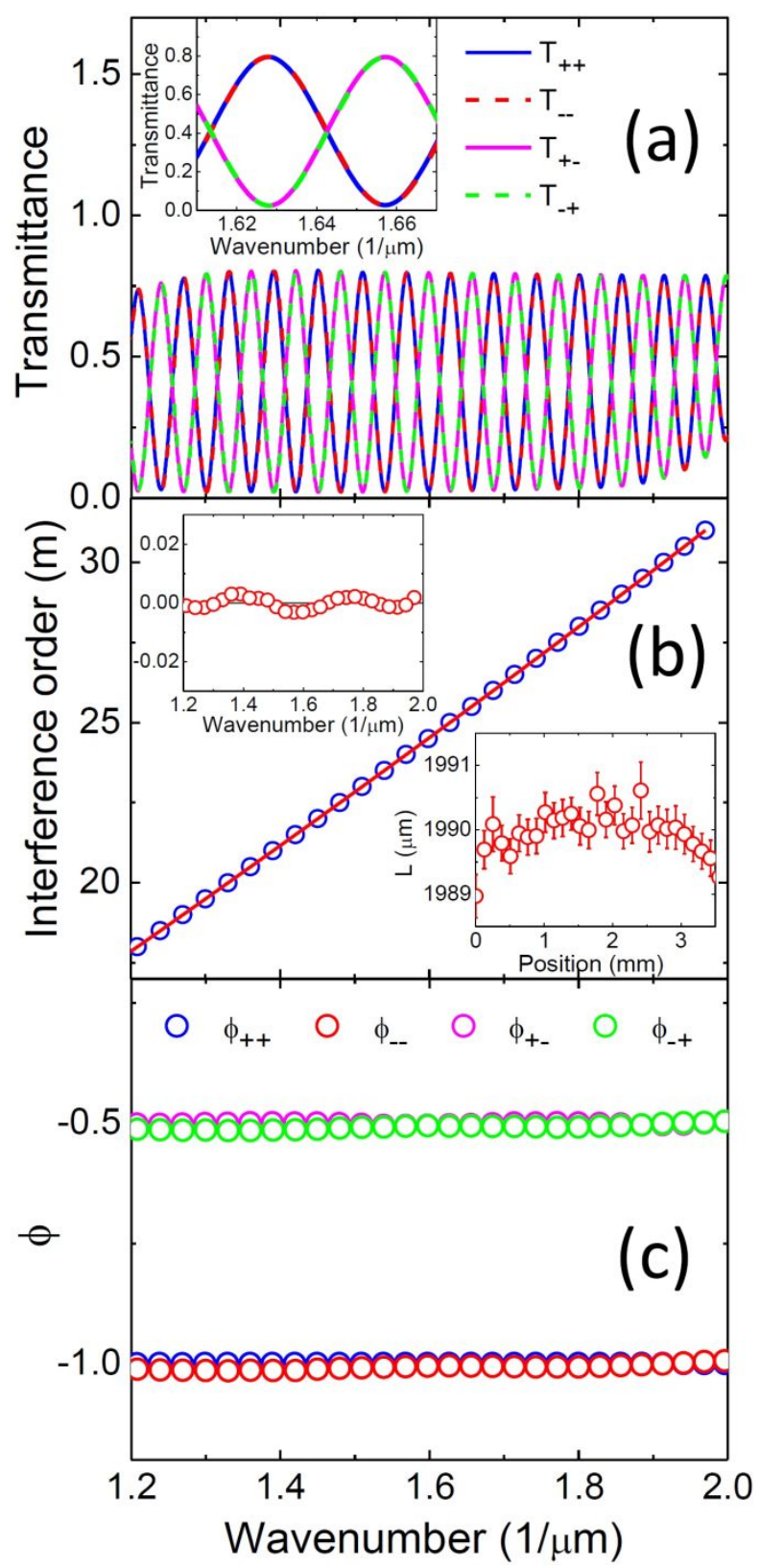

Figure S2. Transmittance and transmission phase of the sapphire substrate. (a) The transmittance of circularly polarized incidence light through an $L=2 \mathrm{~mm}$ thick a-cut sapphire slab with the optical axis as the reference direction using a 20x objective: solid blue, dashed red, solid magenta, and green dashed curves are for,,++--+- , and -+ transmission, respectively. The upper-left inset is the transmittance on 
an expanded scale for about one period. (b) Interference order $m$ vs the corresponding wavenumber $1 / \lambda_{m}$ for transmittance $T_{++}$in (a). The open blue circles are the data and the solid red line is a linear fit to the data using Eq. S12. The left-top inset shows the residual of the fit. The bottom-right inset shows the measured sapphire thickness at various positions along a chosen path using a $5 \mathrm{X}$ objective. (c) Transmission phase of sapphire.

Figure S2a shows the transmittance of circularly polarization incidence $\left(T_{i j}^{S a}\right)$ through a $2 \mathrm{~mm}$ thick a-cut sapphire slab. The transmittance $T_{i j}^{S a}$ exhibit large BI oscillations with $T_{++}^{S a}=T_{--}^{S a}$ and $T_{+-}^{S a}=T_{-+}^{S a}$ (see the inset in Fig. S2a), in good agreement with Eq. S4. In general, the experimental transmission BI oscillations can be represented by [6]:

$$
T_{i j}^{S a}=A+B \cos \left(\frac{2 \pi}{\lambda}\left(n_{o}-n_{e}\right) L+\phi_{i j}^{S a}\right),
$$

where $A$ and $B$ are slowly varying functions of the wavenumber due to the dispersion of the sapphire, and $\phi_{i j}^{S a}=0, \pm \pi$ is the transmission phase of the sapphire. Eq. S10 exhibits transmission peaks for constructive interference satisfying the condition:

$$
\frac{2 \pi}{\lambda_{m}}\left(n_{o}-n_{e}\right) L+\phi_{i j}^{S a}=2 m \pi
$$

with $\lambda m$ the wavelength of the $m^{\text {th }}$ interference order. Eq. S11 can be rewritten with the phase normalized by $2 \pi$ as:

$$
m=\frac{1}{\lambda_{m}}\left(n_{o}-n_{e}\right) L+\phi_{i j}^{S a}
$$

(For simplicity, the $2 \pi$ is dropped for the normalized phase.) Thus, a plot of $m$ vs. $1 / \lambda_{m}$ will give a straight line with a slope equals to $\left(n_{o}-n_{e}\right) L$. Figure $\mathrm{S} 2 \mathrm{~b}$ shows a linear fit (red line) to the $2 \mathrm{~mm}$ sapphire transmission data (blue circles) with $L$ and $\phi_{i j}^{S a}$ as fitting parameters using values of $n_{o}$ and $n_{e}$ from Ref. [3]. The fit is excellent as shown in the residual in the top-left inset. The lower-right inset shows the thickness $(L)$ of the sapphire measured over a long distance showing good uniformity of the sapphire plate. Once $L$ is known, the transmission phase of the 2D Au sawtooth metasurface can then be evaluated at the peaks and troughs by: 


$$
\phi_{i j}^{S a}=m-\frac{1}{\lambda_{m}}\left(n_{o}-n_{e}\right) L,(\mathrm{~S} 13 \mathrm{a})
$$

and

$$
\phi_{i j}^{S a}=m-\frac{1}{\lambda_{m}}\left(n_{o}-n_{e}\right) L+0.5
$$

respectively. The normalized phase is now defined in the range of $[-1,0]$. (Note that there is an additional phase due to the numerical aperture (NA) effect of the microscopic objective [7]. However, as we are interested in the relative phase between LCP and RCP incidence, the phase shift due to the NA effect will be canceled out.) Figure S2c shows the transmission phase for circularly polarization incidence for the $2 \mathrm{~mm}$ sapphire slab. It is clear the co-polarization phases are the same and are $\pi$ out of phase from that of the cross-polarization phases, in good agreement with Eq. S4. Note that Eqs. S13a and S13b can also be used to obtain the transmission phase, hence the circular phase-dichroism, for the Au film as well as the $\mathbf{V}$-, $\mathbf{N}$ -

and $\boldsymbol{И}$-type Au sawtooth metasurfaces.

\section{Transmission dependence on the thickness of the sapphire substrate}

To study the transmission dependence on the thickness of the sapphire plate, instead of fabricating Au sawtooth metasurface on sapphire substrate with various thickness, we place the original $2 \mathrm{~mm}$ sapphire metasurface sample, with index matching, on the top of a $3 \mathrm{~mm}$ thick sapphire plate with its optical axis either parallel or perpendicular to the optical axis of the $2 \mathrm{~mm}$ sapphire substrate to produce, effectively, a total thickness as the sum or the difference of the two sapphire plates. The left/right column of Fig. S3 shows the results for metasurfaces with an effective sapphire thickness of $1 \mathrm{~mm} / 5 \mathrm{~mm}$. It is obvious that the thicker the sapphire substrate, the more the BI oscillations, in accord with Eq. S4 stated above. The insets in Fig. S3 show the transmittance of about one BI oscillation. It is clear that they show the same behavior over one 
period, independent of the thickness of the sapphire substrate. Figure S4 shows the corresponding results from simulations of the metasurfaces, in good qualitative agreement with the experiments.

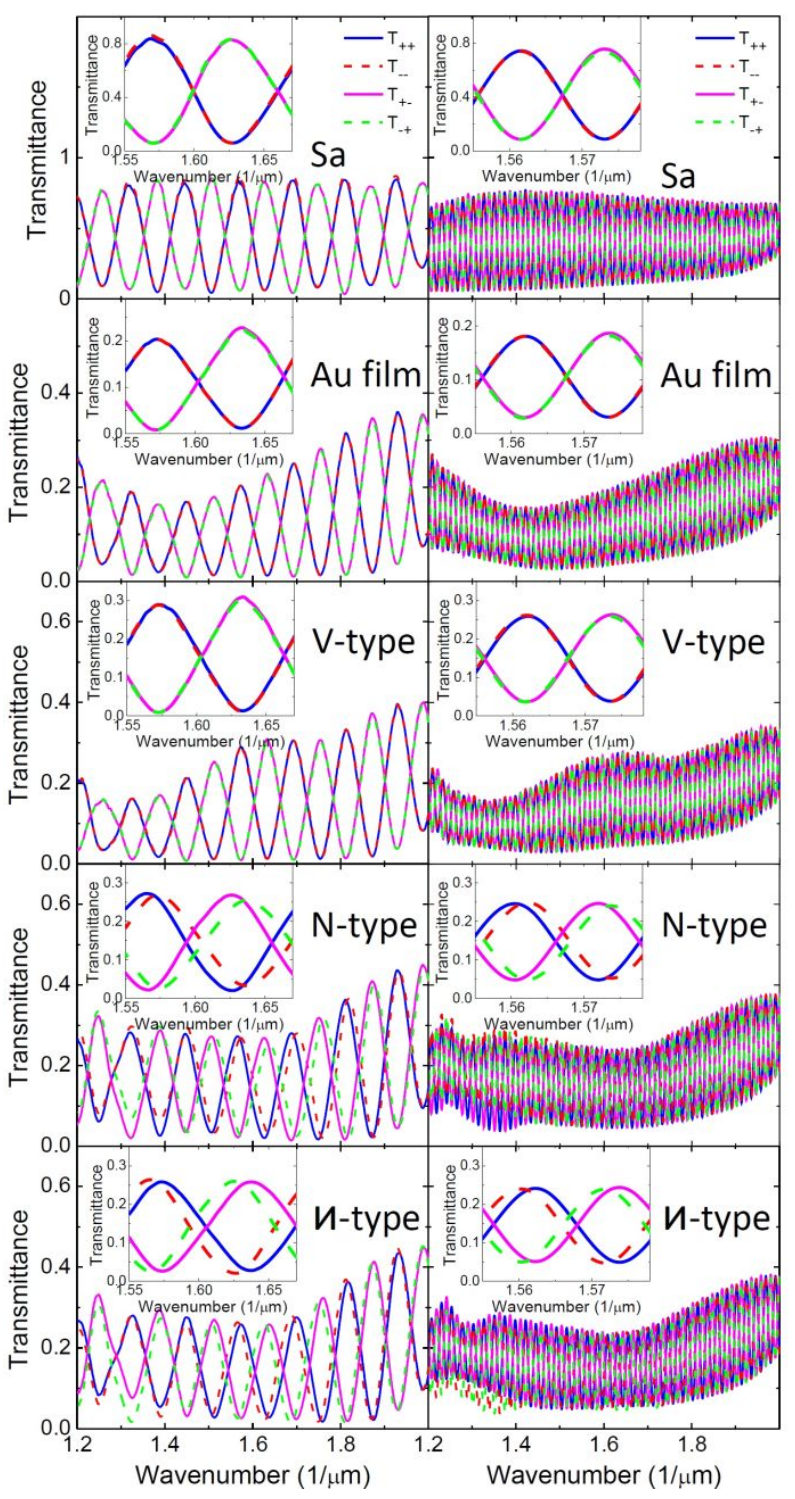

Figure S3. Transmittance measurement of the metasurface. The transmittance of circularly polarized incidence light through sapphire (Sa), Au film, and Au sawtooth metasurface samples, shown in Fig. 2, placed on the top of a $3 \mathrm{~mm}$ thick a-cut sapphire plate to produce effective thickness $L=1 \mathrm{~mm}$ (left column) and 5mm (right column) substrates, respectively. The insets are the transmittance on an expanded scale for about one BI period. 


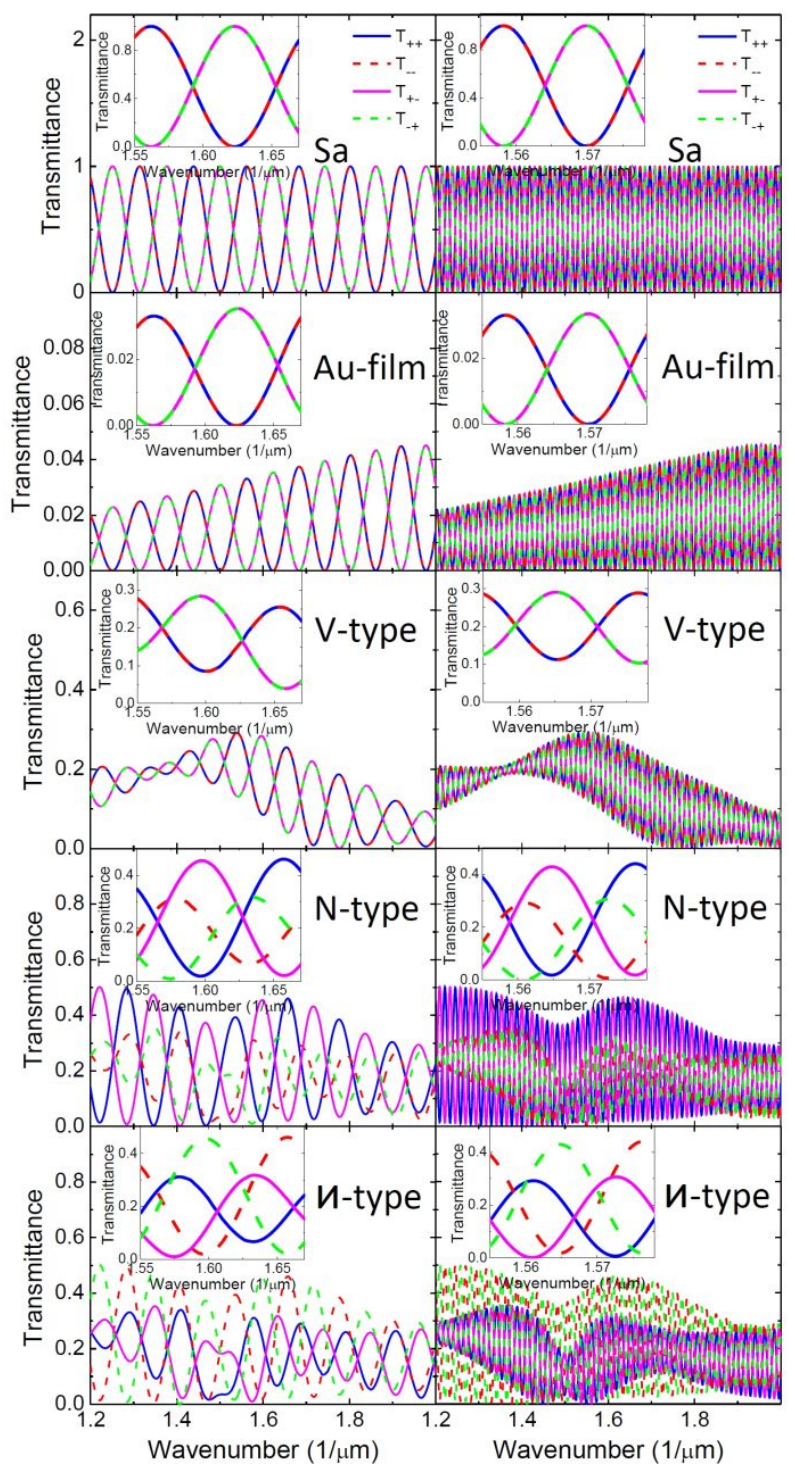

Figure S4. Transmittance from the simulation of 2D Au metasurface. Simulated transmittance of circularly polarized incidence light through sapphire ( $\mathrm{Sa}$ ), Au film, and Au sawtooth metasurface samples in Fig. 3 placed on the top of a $3 \mathrm{~mm}$ thick a-cut sapphire plate to produce effective thickness $L$ $=1 \mathrm{~mm}$ (left column) and $5 \mathrm{~mm}$ (right column) substrates, respectively. The insets are the transmittance on an expanded scale for about one BI period.

Figures S5a and S5b show, respectively, the co- and cross-polarization circular phasedichroism obtained by using Eqs. S9 and S13 for the metasurfaces on effective sapphire thickness $L=1 \mathrm{~mm}$ and $5 \mathrm{~mm}$. The results are almost the same, independent of the substrate thickness, except that there are more data points for the $L=5 \mathrm{~mm}$ sapphire metasurface. Figures 
$\mathrm{S} 5 \mathrm{c}$ and S5d show the corresponding results from simulations of the metasurfaces. They show good qualitative agreement with the experiments.

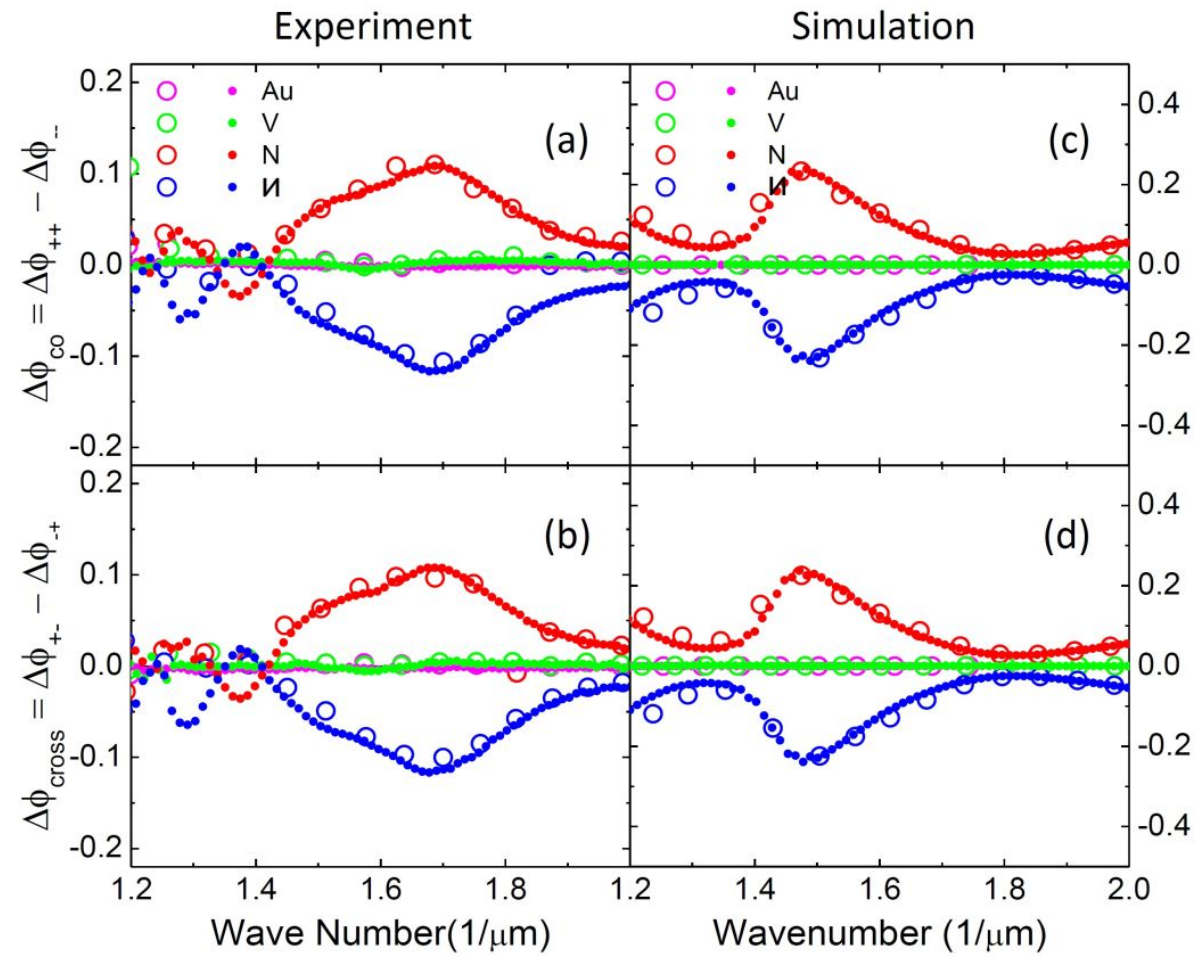

Figure S5. Circular phase-dichroism of the metasurface. (a)-(b) Experimental circular phase-dichroism for $\mathrm{Au}$ film and $\mathrm{Au}$ sawtooth metasurfaces. (c) - (d) Corresponding circular phase-dichroism from simulations. Open and solid symbols are for sapphire thickness $L=1 \mathrm{~mm}$ and $5 \mathrm{~mm}$, respectively.

\section{Circular phase-dichroism by the shift of BI oscillations}

It is clear from the insets of Figs. S3 and S4 that the BI oscillations display relative shifts in the transmittance for the $\mathbf{N}$ - and $\mathbf{U}$-type 2D Au sawtooth metasurfaces w.r.t. that of the sapphire, suggesting that the shift of the peak position could be used in the characterization of the $\mathrm{Au}$ sawtooth metasurfaces. We thus define another chiral parameter based on the shift of the peak position w.r.t. that of the sapphire as:

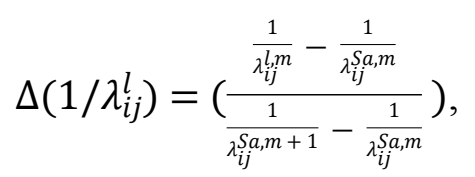


where $1 / \lambda_{i j}^{l, m}$ is the $\mathrm{m}^{\text {th }}$ order peak wavenumber of the $l$-type metasurface and $1 / \lambda_{i j}^{S a, m}$ the corresponding peak wavenumber for the sapphire. Importantly, the circular phase-dichroism based on the BI peak position shift can then be defined for co- and cross-polarization as:

$$
\begin{gathered}
\Delta\left(1 / \lambda_{\text {co }}\right)=\Delta\left(1 / \lambda_{++}^{l}\right)-\Delta\left(1 / \lambda_{--}^{l}\right), \\
\Delta\left(1 / \lambda_{\text {cross }}\right)=\Delta\left(1 / \lambda_{+-}^{l}\right)-\Delta\left(1 / \lambda_{-+}^{l}\right) .
\end{gathered}
$$

Note that $\Delta\left(1 / \lambda_{i j}^{l}\right)$ is also defined in the range of $[-1,0]$, similar to the transmission phase defined in Eq. S9. Furthermore, Eq. S14 can be rewritten using Eq. S12 as:

$$
\begin{gathered}
\Delta\left(\frac{1}{\lambda_{i j}^{l}}\right)=\left(\frac{\frac{1}{\lambda_{i j}^{l, m}}-\frac{1}{\lambda_{i j}^{S a, m}}}{\frac{1}{\lambda_{i j}^{S a, m+1}}-\frac{1}{\lambda_{i j}^{S a, m}}}\right)=\frac{\left(\frac{m-\phi_{i j}^{l}}{\left(n_{o}-n_{e}\right) L}\right)-\left(\frac{m-\phi_{i j}^{S a}}{\left(n_{o}-n_{e}\right) L}\right)}{\frac{1}{\lambda_{i j}^{S a, m+1}}-\frac{1}{\lambda_{i j}^{S a, m}}=\frac{\frac{\phi_{i j}^{S a}-\phi_{i j}^{l}}{\left(n_{o}-n_{e}\right) L}}{\frac{1}{\lambda_{i j}^{S a, m+1}}-\frac{1}{\lambda_{i j}^{S a, m}}}} \\
=\phi_{i j}^{S a}-\phi_{i j}^{l}=-\Delta \phi_{i j}^{l}, \quad \text { (S16) }
\end{gathered}
$$

for $\frac{1}{\lambda_{i j}^{S a, m+1}}-\frac{1}{\lambda_{i j}^{S a, m}}=\frac{1}{\left(n_{o}-n_{e}\right) L}$. Thus the chiral parameter defined by the shift of the peak position is opposite to that defined by the transmission phase. 


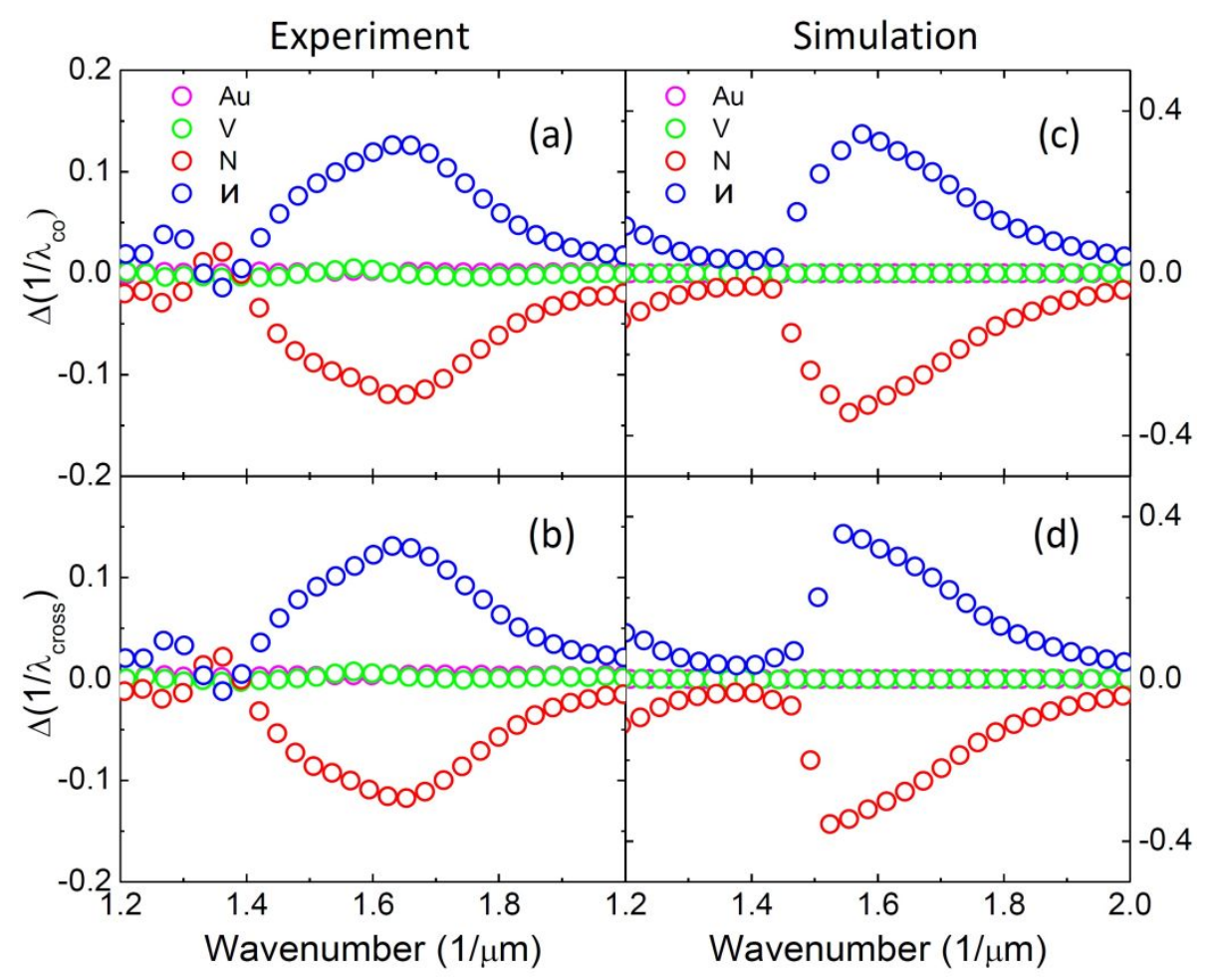

Figure S6. Chiral parameter of metasurface based on peak shift. (a)-(b) Experimental chiral parameter based on the shift of wavenumber w.r.t. that of the sapphire for Au film and 2D Au sawtooth metasurfaces. (c)-(d) Corresponding chiral parameter from simulations. The thickness of the sapphire substrate is $L=2 \mathrm{~mm}$.

Figure S6 shows the results of the chiral parameter based on the shift of the BI peaks for the metasurfaces shown in Fig. 4 with $L=2 \mathrm{~mm}$ sapphire substrate. The results look very similar, except the sign, to the results obtained by fitting of the interference order vs the wavenumber as given by Eq. S13 for the circular phase-dichroism based $\Delta\left(\phi_{i j}^{l}\right)$ as $\Delta\left(\frac{1}{\lambda_{i j}^{l}}\right)$ given by Eq. S14 is equal to $-\Delta\left(\phi_{i j}^{l}\right)$ as shown in Eq. S16.

\section{Non-birefringent substrate metasurface}

To investigate the role of the substrate, we also fabricated 2D Au sawtooth nanoarray metasurfaces on $1 \mathrm{~mm}$ thick ITO glass by using an e-beam direct write and a lift-off process as reported earlier [1]. The left column of Fig. S7 shows the transmittance of the ITO Au sawtooth 
metasurfaces for circularly incident light with the Au pattern facing upward and illuminated from below. The insets show the SEM image of the unit cells of the metasurfaces as labeled. Note that the ITO 2D Au sawtooth nanoarrays are more rounded as compared to the sapphire Au sawtooth nanoarrays shown in Fig. 1 due possibly to different fabrication methods. Importantly, the transmittance for the Au film and the 2D V-type sawtooth metasurface are the same for both the co- and cross-polarization while they are different for the $\mathbf{N}$ - and $\boldsymbol{U}$-type $\mathrm{Au}$ sawtooth metasurfaces. (The small difference at wavenumber 1.2-1.3 $(1 / \mu \mathrm{m})$ range could be due to the leakages of the polarizers/quarter waveplates and the imperfections of the metasurfaces.) Overall, this is in accord with 2D chiral materials in which the transmissions for the co-polarization are equal, i.e. $T_{++}^{l}=T_{--}^{l}$, but different for the cross-polarization, i.e. $T_{+-}^{l} \neq T_{-+}^{l}$, due to the polarization conversion of the metasurfaces [1].

In order to obtain the transmission phase of the metasurface on the ITO glass substrate, we place the ITO samples on the top of a $2 \mathrm{~mm}$ thick sapphire as shown in the top inset. (For metasurface with an absorptive substrate, instead of transmission it would be better to put the sapphire plate onto the sample side for reflection phase measurements.) The middle column in Fig. S7 shows the transmittance of the ITO samples with the additional sapphire plate. Note that the $\mathbf{N} / \mathbf{M}$-type sawtooth metasurface is the same sample as the $\mathbf{N} / \mathbf{U}$-type sawtooth metasurface in the left column. BI transmission oscillations are now observed, similar to those of the sawtooth metasurfaces fabricated directly on sapphire substrates. The insets show the BI oscillations for about one period, showing clearly similar transmittance shifts as those produced by the metasurfaces fabricated directly on sapphire substrates. The right column of Fig. S7 shows the transmission phase w.r.t. that of the sapphire obtained from the fits to the interference 
order vs the peak wavenumber data as explained above. It is clear that the $\mathbf{N}$ - and $\mathbf{M}$-type metasurfaces exhibit opposite chiral property, consistent with the metasurfaces on sapphire substrates.

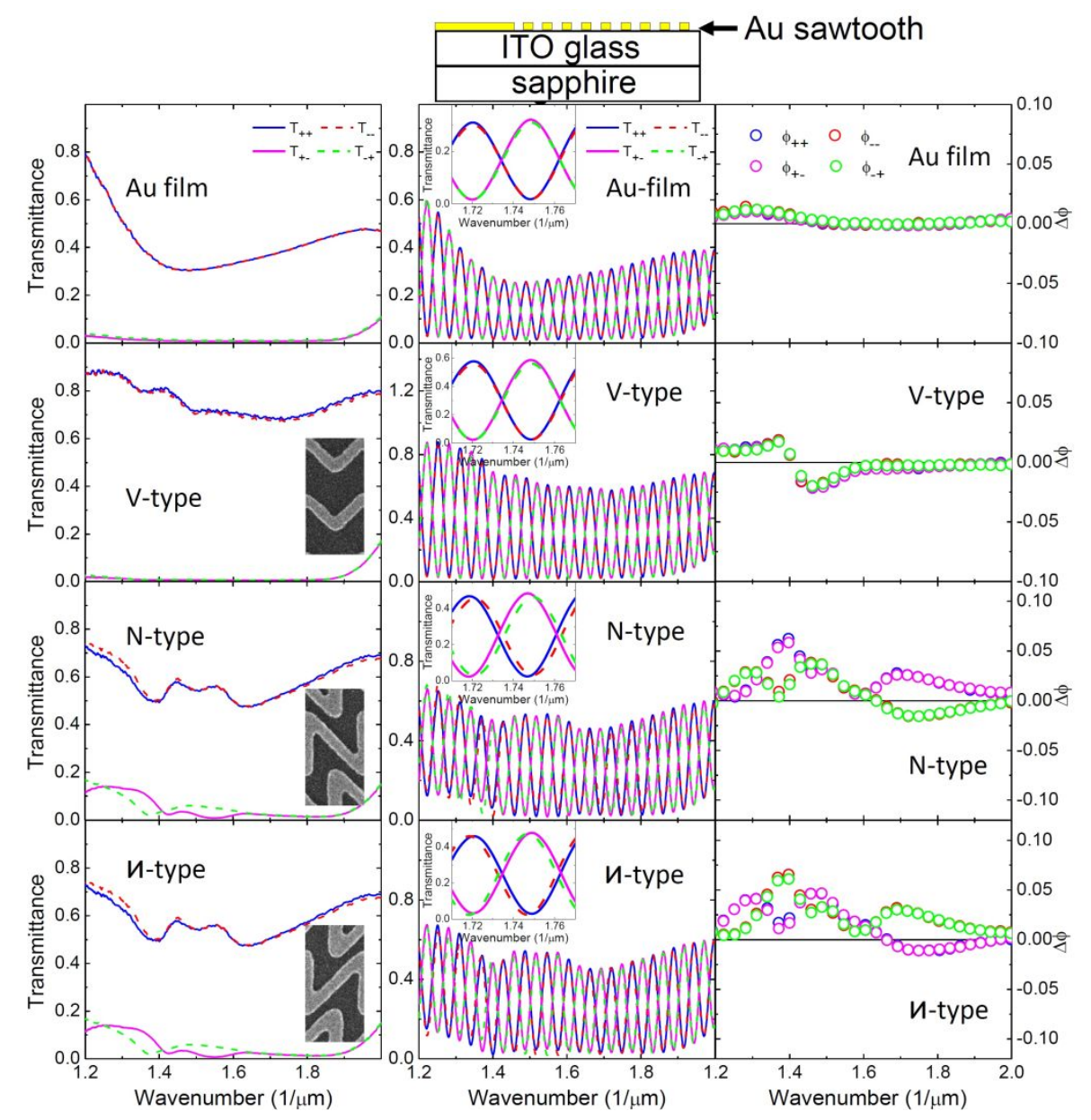

Figure S7. Transmittance and phase of metasurface of the non-birefringent substrate. Left column transmittance of circularly polarized incidence light of $\mathrm{Au}$ film and $\mathrm{Au}$ sawtooth metasurfaces on ITO glass. Insets are SEM images of unit cells for the metasurfaces. Middle column - transmittance of Au film and Au sawtooth metasurfaces on ITO glass placed, with matching fluid, on the top of an $L=2 \mathrm{~mm}$ thick a-cut sapphire as shown in the top inset. The insets are the transmittance on an expanded scale for about one BI period. Right column - open circles are corresponding transmission phase w.r.t. sapphire obtained from Eq. S13 as labeled for the Au film, $\mathbf{V}$-type, $\mathbf{N}$-type, and $\boldsymbol{U}$-type sawtooth metasurfaces, respectively. The Au film and the Au sawtooth arrays are 30nm thick.

Figures S8a and S8b show the co- and cross-polarization circular phase-dichroism for the ITO metasurfaces placed on the sapphire plate. The results are consistent with those 
of the metasurfaces fabricated directly on sapphire plates. For comparison, the conventional co- and cross-polarization circular dichroism (CD) are also shown in Figs. S8c and S8d, respectively. The $\mathbf{N}$ - and $\boldsymbol{И}$-type ITO metasurfaces exhibit reasonable CD in the $\sim 1.2-1.6(1 / \mu \mathrm{m})$ wavenumber range for the cross-polarization but not for the copolarization. Note that the CDs for the $\mathbf{N}$ - and $\boldsymbol{И}$-type ITO metasurfaces are non-zero around the 1.2-1.4 $(1 / \mu \mathrm{m})$ wavenumber range for the co-polarization due possibly to the imperfections of the polarizers and quarter waveplates, in addition to the quasi-three dimensional nature of the samples. Figure S8 shows clearly that the CPD exhibits similar sensitivity as the conventional CD supporting strongly that the CPD is a good alternative and supplements the CD measurement in the characterization of chiral materials.

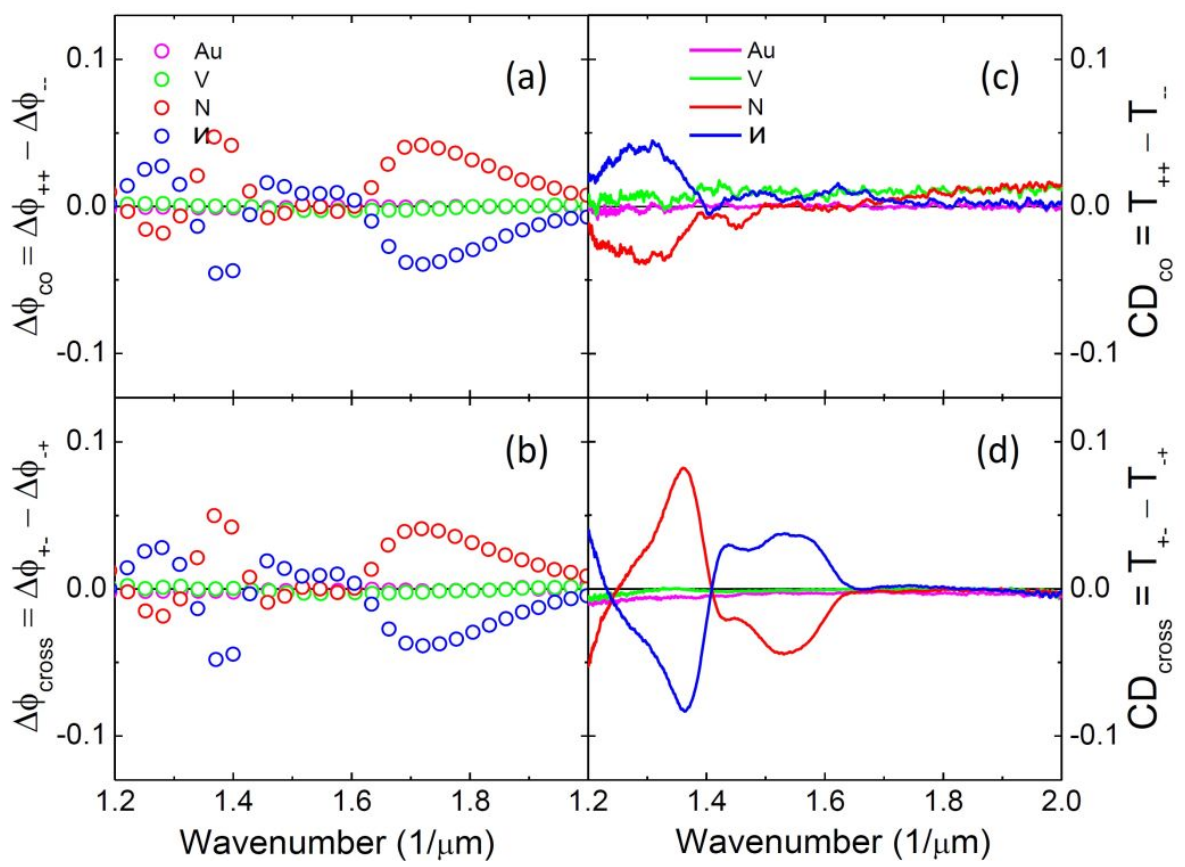

Figure S8. Circular phase-dichroism of metasurface with the non-birefringent substrate. (a)-(b) Experimental circular phase-dichroism for Au film (magenta), and $\mathbf{V}$-type (green), $\mathbf{N}$-type (red) and $\boldsymbol{U}$ type (blue) sawtooth metasurfaces on $1 \mathrm{~mm}$ ITO glass placed on the top of an $L=2 \mathrm{~mm}$ thick a-cut sapphire. (c)-(d) Corresponding CD of the sawtooth metasurfaces in (a)-(b). 


\section{Comparison of the CPD and CD measurements}

In order to compare further the sensitivity of the CPD and the conventional CD in the characterization of chiral metasurfaces, we performed more measurements and analysis using the samples used in Fig. 2a. Note that when the thickness of the sapphire substrate is zero, i.e. $L=0$, the BI oscillations will disappear and the transmittance given by Eq. 2 can then be used to calculate the conventional $\mathrm{CD}$. This can be achieved by placing the original metasurface sample fabricated on a sapphire substrate on the top of another matching thickness sapphire plate with an optical axis orthogonal to that of the sample substrate to create, effectively, a zero substrate thickness (zero-thickness method) as shown schematically on the top inset of the left column in Fig. S9. The left column of Fig. S9 shows the transmittance for the Au film and the sawtooth metasurfaces used in Fig. 2a placed on a $2 \mathrm{~mm}$ thick sapphire plate with an optical axis orthogonal to that of the original sapphire substrate. It is clear that the BI oscillations observed in Fig. 2a now disappear with only a very small remnant effect due to the non-perfect matching of the sapphire thickness. Note that the transmittance for the different incident and detection polarizations are the same for the achiral $\mathrm{Au}$ film and the $\mathbf{V}$-sawtooth metasurface while they show complementary behavior for the chiral $\mathbf{N}$ - and $\boldsymbol{U}$-type sawtooth metasurfaces.

In addition to the zero-thickness method mentioned in above, it turns out that the original BI oscillations obtained in Fig. 2a can also be used to obtain the conventional CD. It is noted that the oscillations due to the birefringent interference are bounded by the maximum and minimum transmittance, corresponding to the transmission measurement in the co- and cross-polarization configurations, of the metasurface without the sapphire substrate. Thus one can approximate 
the co- $\left(T_{++}^{l}\right.$ and $\left.T_{--}^{l}\right)$ and the cross- $\left(T_{+-}^{l}\right.$ and $\left.T_{-+}^{l}\right)$ transmittance by the peak and trough values of the BI oscillations (envelope method).

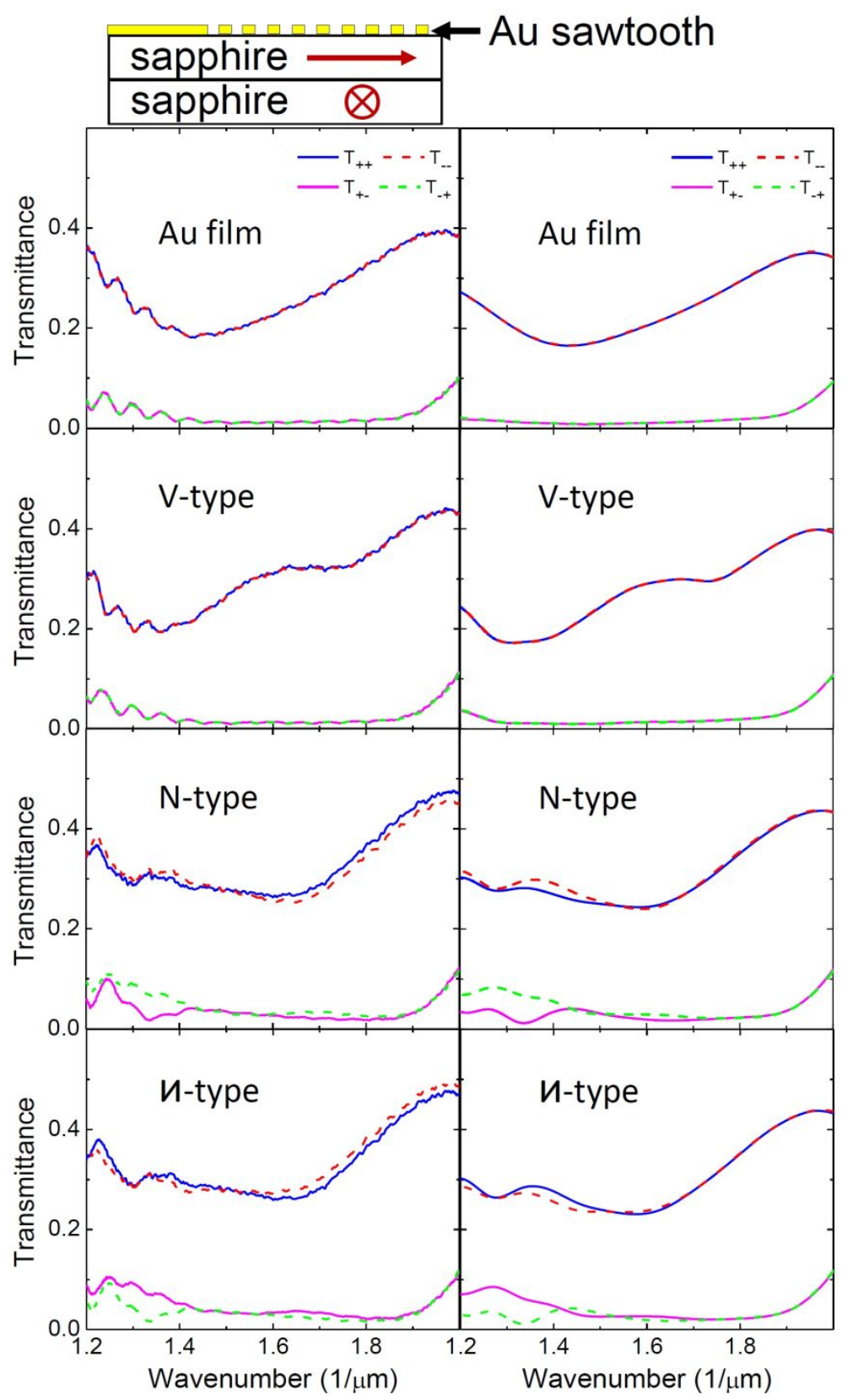

Figure S9. Left column - transmittance of the Au film and Au sawtooth metasurfaces shown in Fig. 2a on a matching sapphire plate with orthogonal optical axes as indicated by the arrows shown in the top inset (zero-thickness method). Right column - corresponding transmittance the Au film and $\mathrm{Au}$ sawtooth metasurfaces using the peak and trough envelopes for the co- and cross-polarizations, respectively, of the experimental data in Fig. 2a (envelope method). 
The right column of Fig. S9 shows the corresponding transmittance for the Au film and sawtooth metasurfaces for the envelope method obtained by fitting the peaks and troughs of the BI oscillations in Fig. 2a. They agree very well with that transmittance obtained by the zero-thickness method shown in the left column of Fig. S9 and confirm the achiral behavior of the Au film and the $\mathbf{V}$-sawtooth metasurface and, importantly, the complementary behavior for the chiral $\mathbf{N}$ - and $\boldsymbol{И}$-type sawtooth metasurfaces for the different incident and detection polarizations.

Figures S10a and S10b show the CDs obtained using the above two methods, zerothickness and envelope, for the co- and cross-polarizations, respectively. The results are consistent with each other considering the scatters of the data due to the non-perfect matching of the sapphire thickness in the zero-thickness method and also the errors in the peak and trough values in the envelope method such that the $\mathrm{CD}$ for the copolarization is very small or practically zero, as expected for $2 \mathrm{D}$ chiral metamaterials, and also the good agreement of the CD response for the two methods at the 1.25-1.4 $(1 / \mu \mathrm{m})$ wavenumber range for the cross-polarization. Note that the maximum $\mathrm{CD}$ is about 0.05 at $1.35(1 / \mu \mathrm{m})$ wavenumber for the cross-polarization. The CPD results shown in Figs 3a and 3b in the main text are replotted as Figs. S10c and S10d for a direct comparison with the $\mathrm{CD}$ measurements. Overall, the CPD signals are much larger than the CD signals. Importantly, the CPD signals are the same for the co- and crosspolarization whereas they are different for the $\mathrm{CD}$ signals. Thus, either the co- or the cross-polarization CPD can be used to characterize metasurfaces, suggesting clearly that the $\mathrm{CPD}$ approach is a more robust approach, if not more sensitive, than the $\mathrm{CD}$ approach. 


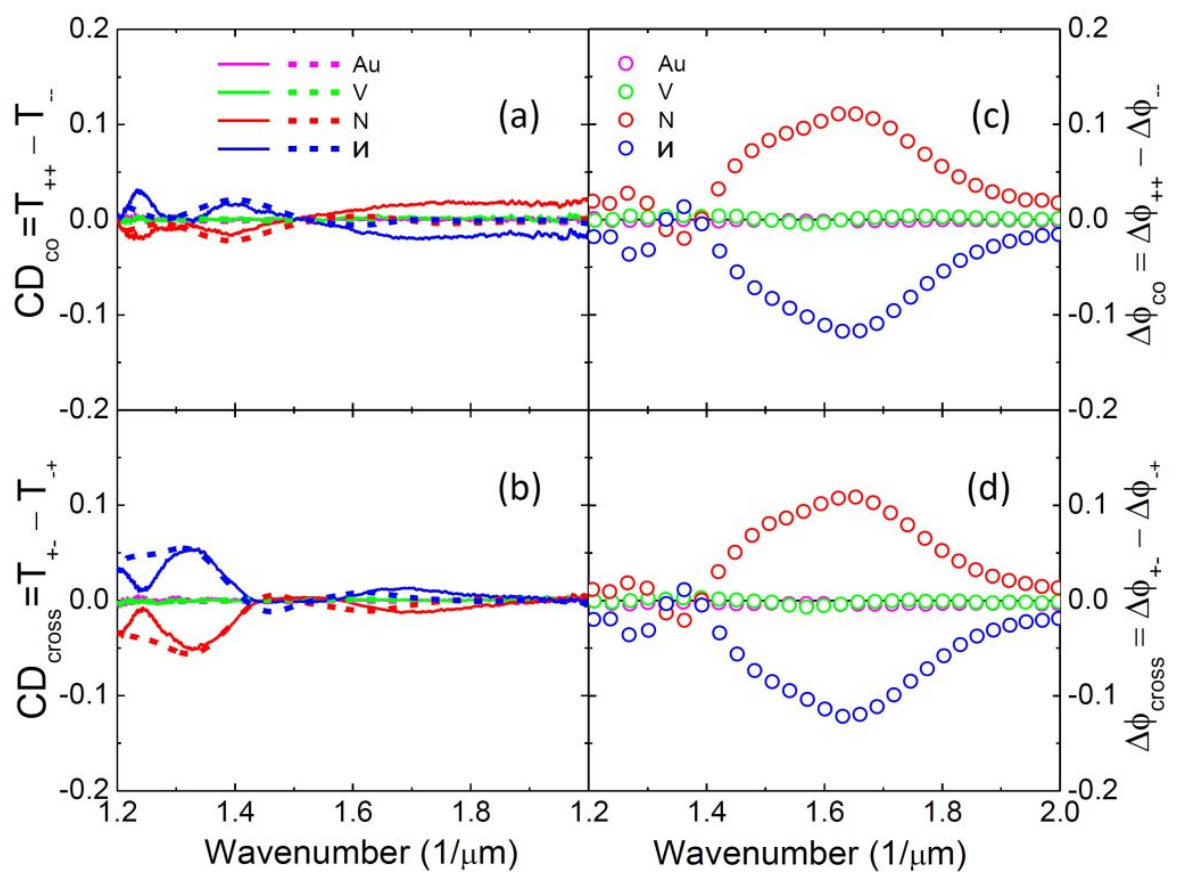

Figure S10. (a) - (b) CD obtained using the transmittance in Fig. S9 for the zero-thickness method (solid curves) and the envelope method (thick dashed curves) of the experimental data in Fig. 2a. (c) - (d) Experimental circular phase-dichroism for Au film and Au sawtooth metasurfaces from Fig. 3.

\section{Reference}

[1] Gao W.; Ng C. Y.; Leung H. M.; Li Y.; Chen H.; Tam W. Y. Circular dichroism in single layered gold sawtooth gratings. JOSA 2012, B29, 3021-3026.

[2] Gao W.; Tam W. Y. Optical Activities in Complementary Double Layers of Six-armed Metallic Gammadion Structures. J. Opt. 2011, 13, 015101.

[3] Harris D. C.; Johnson L. F.; Cambrea L.; Baldwin L.; Baronowski M.; Zelmon D. E.; Poston W. B.; Kunkel J. D.; Parish M.; Pascucci M. R.; Gannon J. J.; Wen Jr. T. C. Refractive index of infrared-transparent polycrystalline alumina. Opt. Eng. 2017, 56(7), 077103.

[4] Yeh P. Extended Jones matrix method. JOSA 1982, 72, 507-513.

[5] Menzel C.; Rockstuhl C.; Lederer F. An advanced Jones calculus for the classification of periodic metamaterials. Phy. Rev. A 2010, 82, 053811.

[6] Yung T. K.; Zhao Q.; Gao W.; Wang X.; Tam W. Y. Measurement of reflection phase using thick-gap Fabry-Perot etalon. App. Opt. 2016, 55, 7301-7306.

[7] Zhao Q.; Yung T.K.; Wang X.; Tam W. Y. Correction of numerical aperture effect on reflection phase measurement using thick-gap Fabry Perot etalon. App. Opt. 2017, 15, 43924397. 